\title{
SPARSE DESCRIPTION OF THE CORTICAL SURFACE PEDIATRIC DEVELOPMENT: A SULCAL PITS STUDY
}

\author{
I. Kaltenmark, L. Brun, G. Auzias, J. Lefèvre, C. Deruelle, O. Coulon \\ Institut de Neurosciences de la Timone, UMR 7289, Aix-Marseille Université, CNRS, Marseille, France.
}

\begin{abstract}
Despite the large variability of sulcal folds, their deepest points, namely the sulcal pits, provide a sparse and reproducible description of the cortical surface. Their role during the antenatal and pediatric development of the brain remains unclear. This work is the first study of the evolution of all sulcal pits during healthy pediatric development. We propose a new method to identify sulcal pits across subjects that benefits from the input of sulcal basins using a varifold metric. We then present a procedure to extract the amplitude and principal direction of sulcal pits trajectories.
\end{abstract}

Index Terms - brain development, cortical folding, sulcal pits, sulcal basins, varifolds

\section{INTRODUCTION}

A major interest of sulcal pits is to define a finite set of robust landmarks on the cortical surface allowing local comparison across individuals. They have been often used to describe adult cortical morphometry [1] or atypical sulcal pattern in pathologies [2] but they have been largely neglected to study the typical post-birth cortical folding. The longitudinal study of [3] on the first two years of life highlighted a stable spatial distribution of pits in the few major deepest folds already present at term birth. Authors also reported a regionally heterogeneous increase of sulcal pits depth, especially in the high-order association cortex. Another study on children between 18 months and 10 years and covering both deep and shallow folds showed that the number of pits increases with age suggesting an increase of the number of folds even after term birth [4], which regarding previous results would likely concerns shallow folds. However, the exact location and time table of these changes remain unexplored, probably because of the lack of comprehensive model to track the position of sulcal pits across subjects and time. Moreover, the high spatial inter-individual variability can hinder the detection of subtle temporal changes.

While a high spatial consistency has been often reported for the deepest sulcal pits, the most shallow pits yet show greater spatial variability across subjects [5]. These shallow pits are also less represented across the population, with a possible phenomenon of spatial pattern modes in the popula- tion. This observation is inherent to the fact that these pits, compared to the deepest ones, are probably under lesser genetic control and rather subject to mechanical influence during the surface folding process.

The identification of homologous pits across subjects, either by clustering after registration $[5,6]$ or graph matching $[7,1]$, is made difficult by the large inter-subject variability. Several studies avoid this issue by considering only the deepest folds (e.g. [3]).

This paper presents a new method to describe the postnatal developmental trajectory of sulcal pits relying on two main contributions. First, we suggest several improvements in the definition of the group-wise labeling by combining information from sulcal pits position and the basins around those pits using a varifold metric. Second, we propose to use an exponential smoothing to extract the spatial trajectory of each labeled pit across time, out of the inter-individual spatial variability. In the following, we detail the methodological improvements of our contributions and we apply this framework to investigate the spatio-temporal trajectory of sulcal pits on linearly aligned brains of 155 children between 9 months and 18 years.

\section{PITS IDENTIFICATION}

\subsection{Data Acquisition}

The Cincinnati MR Imaging of Neurodevelopment (C-MIND) project provides MRI data of 209 healthy children between 0 and 18-years-old (04/30/2015 release), among them 59 subjects were scanned longitudinally with two or three timesteps. For each timestep, one or two T1w images were acquired on a Philips 3T MRI scanner with the following protocol: matrix size $=256 \times 224$, spatial resolution $=1 \mathrm{~mm} \times 1 \mathrm{~mm} \times 1 \mathrm{~mm}$, $\mathrm{TR} / \mathrm{TE}=8.1 / 3.7 \mathrm{~ms}$, flip angle $=8, \mathrm{TI}=939 \mathrm{~ms}$. These images were denoised using SPM8 (SANLM algorithm) and a grey/white matter segmentation was performed using FreeSurfer 5.3. We selected 155 subjects from the Cincinnati Childrens Hospital Medical Center study site, ages between 9 months and 18 years, whose images were properly segmented and grey-white interface correctly extracted. Among them, 10 subjects were manually corrected using the control points of FreeSurfer. 


\subsection{Image Processing, Cortical Surface and Sulcal Pits Extraction}

The extraction of sulcal pits was performed using the procedure of [5], designed to yield reproducible sulcal pits especially in children populations. For each subject, we first estimated the sulcal depth map using the depth potential function, a measure that integrates both curvature and convexity information [8]. Then, we applied a watershed by flooding algorithm that computes the sulcal basin associated to each depth maximum of the cortical surface and provides a complete parcellation of this surface. A merging of sulcal basins was performed during the flooding in order to filter spurious extrema caused by noise in the depth map. Filtering parameters were optimized as described in [5]. We obtained a set of $97 \pm 6.9$ pits by subject, increasing with age.

\subsection{Pits Clustering}

The next step consists in defining a group-level clusters of pits that is usually computed by a watershed algorithm on the density map of the pits positions after spherical alignment of the meshes on a smooth atlas surface $[3,5]$. We chose to use a preexisting cluster map [5] with 108 regions of an adult population to ensure its stability.

According to our best knowledge, the reproducibility of the sulcal basins has not been studied and yet seems significant. The regions defined by the cluster map could thus be seen as an atlas of sulcal basins. In the literature, the label of a pit (affectation to its cluster) is estimated by a simple projection on the atlas surface (Method 1) [3, 5]. However, if one considers now the cluster map as an atlas of basins, then the global registration of the surface does not always perform a good registration of the basins and the pit of a subject can fall close to the boundary of an atlas basin, questioning its affectation. With our population, we count $8.2 \%$ of pits that are projected on the boundary of an atlas basin and $15.3 \%$ are very close to the boundary (in a two-ring neighborhood).

\subsection{Pits Labeling}

For this study, we propose a new method, Method 2, to assign a subject pit to an atlas basin. With the procedure of [5], subject basins and template basins define respectively a complete parcellation of the subject cortical surface and the atlas surface. The central idea is to compare registered subject basins to atlas basins with a similarity measure $\mathcal{M}$ on surfaces. For this purpose, we used an oriented varifold metric which compare positions, areas, and orientations of the surfaces $[9,10]$. This new method relies thus on the reproducibility of the sulcal basins. Observations of the data showed that this hypothesis is yet patently not satisfied in one situation: a subject misses pits in a region and one of its basin covers thus one or more atlas basins. For this reason, we define an algorithm that relies either on the pit or the basin of the subject. Atlas basins are ordered by the group-watershed algorithm: most reproducible basins are in the top of this list denoted $\bar{Q}$. As the number of pits varies across individuals, our method might compensate impossible pairing with the second best option. To prevent this situation, we add a reject criteria grounded on the overlap between two paired basins. We denote $B \cap \bar{B}>P \% \bar{B}$ if the intersection of two basins $B$ and $\bar{B}$ fills at least $P \%$ of $\bar{B}$. The overlap criteria ensures that if a pit is not assigned to its parent cluster, its cluster does overlap subsequently with the pit's basin. Given a registered subject:

1. Order subject basins by decreasing area in a queue $Q$. For each basin $B \in Q$, consider the atlas basin $\bar{B}$ that contains the subject pit. (a) If $B \cap \bar{B}>80 \% \bar{B}$, the pit is assigned to $\bar{B}$ and $\bar{B}$ is removed of $\bar{Q}$. (b) If the area of $B$ is twice larger than the area of $\bar{B}$, and if $B \cap \bar{B}>50 \% \bar{B}$, the pit is assigned to $\bar{B}$ and $\bar{B}$ is removed of $\bar{Q}$.

2. For each atlas basin $\bar{B} \in \bar{Q}$, ordered unassigned subject basins in a new list $Q^{\prime}$ by decreasing distance to $\bar{B}$ with respect to the similarity measure $\mathcal{M}$. Select the first subject basin $B \in Q^{\prime}$ that satisfies the overlap criteria: either $B \cap \bar{B}>50 \% \bar{B}$ or $B \cap \bar{B}>50 \% B$.

With the natural cluster assignation, Method 1, if several pits fall in the same cluster, the deepest one is kept, the others are unassigned. Our algorithm prevents any multiple assignments.

\subsection{Optimization of the atlas basins}

To quantify the reproducibility of pits for each atlas basin, we computed the percentage of subjects who have one and only one pit assigned to this basin, called the $N_{1}$ measure [5]. At the individual level, the sulcal basins generated by the watershed algorithm from the depth potential map fit the geometry of the cortical surface. At the group level however, the density map induces a bias on the atlas basins, overextending the clusters of the most represented pits at the expense of shallow pits. We therefore optimized the atlas basins after a first run of our method with a weighted voting strategy. For each vertex of the atlas surface, we computed among the projection of subject basins the occurrence of each label weighted by the $N_{1}$ value of the label. This provides a new atlas parcellation for which we apply again our labeling method.

\section{PITS TRAJECTORIES}

Labeling the pits allows us to return to the baseline surfaces and follow the evolution of the pits across the population for each label independently. All subject surfaces are aligned with a rigid registration on a reference surface chosen as the subject surface that minimizes the distance to its closest 
neighbors and whose age is close to the mean of the population. We then regroup the pits among the whole population according to their label. Each pit is given as a point in the 3D-space with a temporal marker that corresponds to the age of the subject from which it is extracted. Hence, for each label, we have a cloud $\left\{t_{i} \mapsto y_{i} \in \mathbb{R}^{3}\right\}_{i=1 . . N}$ of $N$ 3D-points $\left(y_{i}\right)$ indexed by age $\left(t_{i}\right)$. To analyze this type of data, we performed an exponential smoothing as follows:

$$
\hat{y}_{i}=\left(\sum_{j=1}^{N} y_{j} \exp ^{-\frac{\left|t_{i}-t_{j}\right|^{2}}{\sigma^{2}}}\right) /\left(\sum_{j=1}^{N} \exp ^{-\frac{\left|t_{i}-t_{j}\right|^{2}}{\sigma^{2}}}\right) .
$$

This filtering aims at reducing the spatial variability of the pits across the population while preserving the temporal variability, as long as the scalar parameter $\sigma$ remains small. In our experiments, $\sigma=1.5$ year. Fig. 1 illustrates this process for one label.

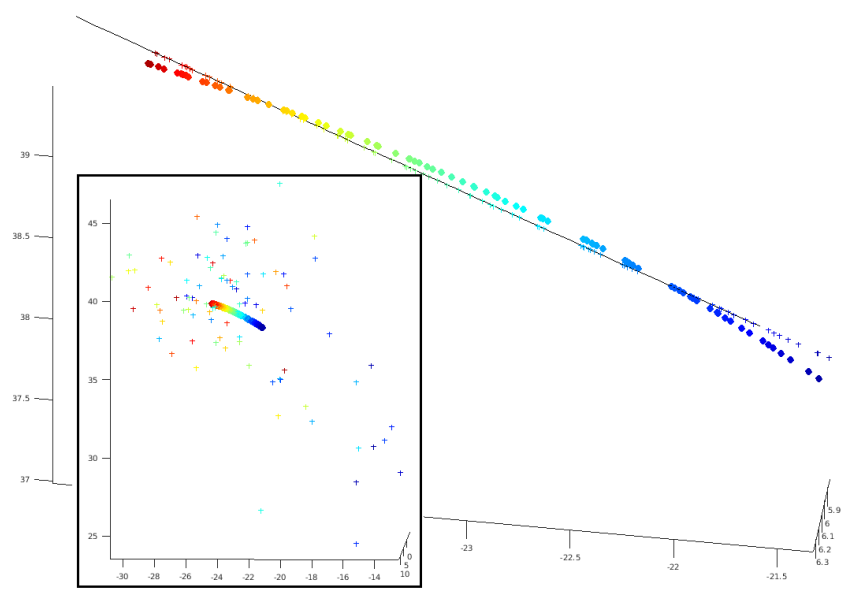

Fig. 1. Illustration of the procedure for one label. Front: Exponential smoothing of the pits. Back: Linear regression and projection of the smoothed pits.

The next step is to perform a linear regression on the smooth pits with respect to age. In most cases, the p-value is highly significant (median at $10^{-26}$ ). However, although the pits may be aligned, the speed of the trajectory with respect to age is not necessarily linear. We projected the trajectory on the image of the linear regression to better fit the data as illustrated in Fig. 1. At last, we extracted the single vector that represents the projection of the pits on this line and hence indicates the main direction and amplitude of temporal development. For the few basins with poor p-value $\left(>10^{-5}\right)$, we visualized the results to ensure that this vector gives a good first order approximation of the trajectory.

The exponential smoothing turns each cloud of pits into a smooth trajectory. In order to ensure that the linear structure is a meaningful description of the pits evolution with respect to time, we perform the following test. For each label, we applied the exponential smoothing for $n=30000$ random permutations of the age index of the pits and we computed the

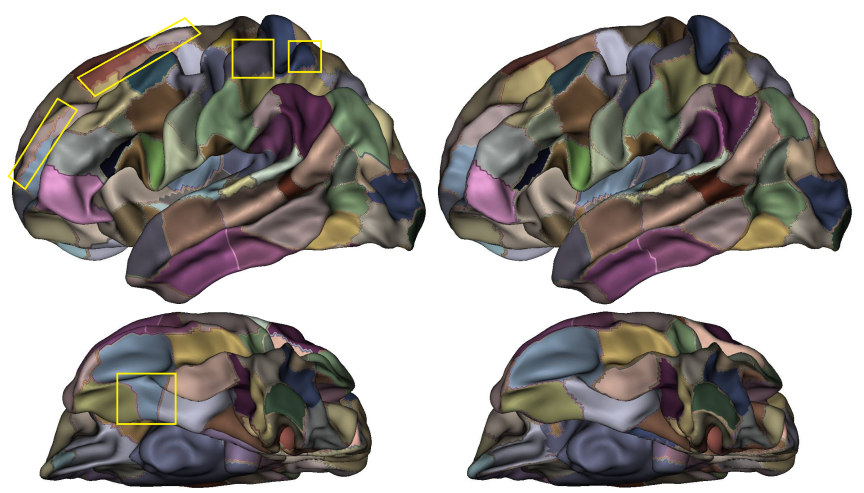

Fig. 2. Optimization of the atlas basins.

ratio of the standard deviation before and after exponential smoothing. The ratio for the original index can then be compared to those obtained by random permutation. If the reduction of the standard deviation is lower than $95 \%$ of the ratios randomly generated, we consider that age is a significant factor to explain the position of the pits. With this threshold, 32 basins are retained.

\section{RESULTS AND DISCUSSION}

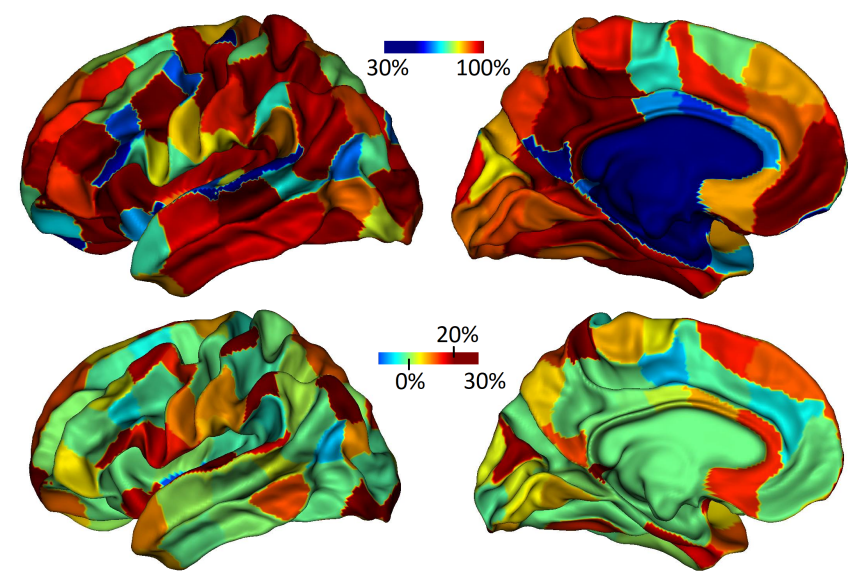

Fig. 3. On the top: $N_{1}$ map for Method 2. On the bottom: $N_{1}$ increase with respect to Method 1.

Fig. 2 compares the atlas basins before and after the voting strategy. One can observe that the new boundaries better fit the geometry of the surface and some artifacts of the initial map are corrected (see in the temporal lobe). The proposed Method 2 labels $85.1 \%$ of the pits against $77.8 \%$ for the Method 1. This increase mostly benefits to shallow pits as displayed in Fig. 3 alongside the $N_{1}$ score for Method 2. Moreover, the two methods attribute different labels to $21.7 \pm 4.9 \%$ of a subject's pits. A visual validation of the results showed that in most conflicting cases our method was 

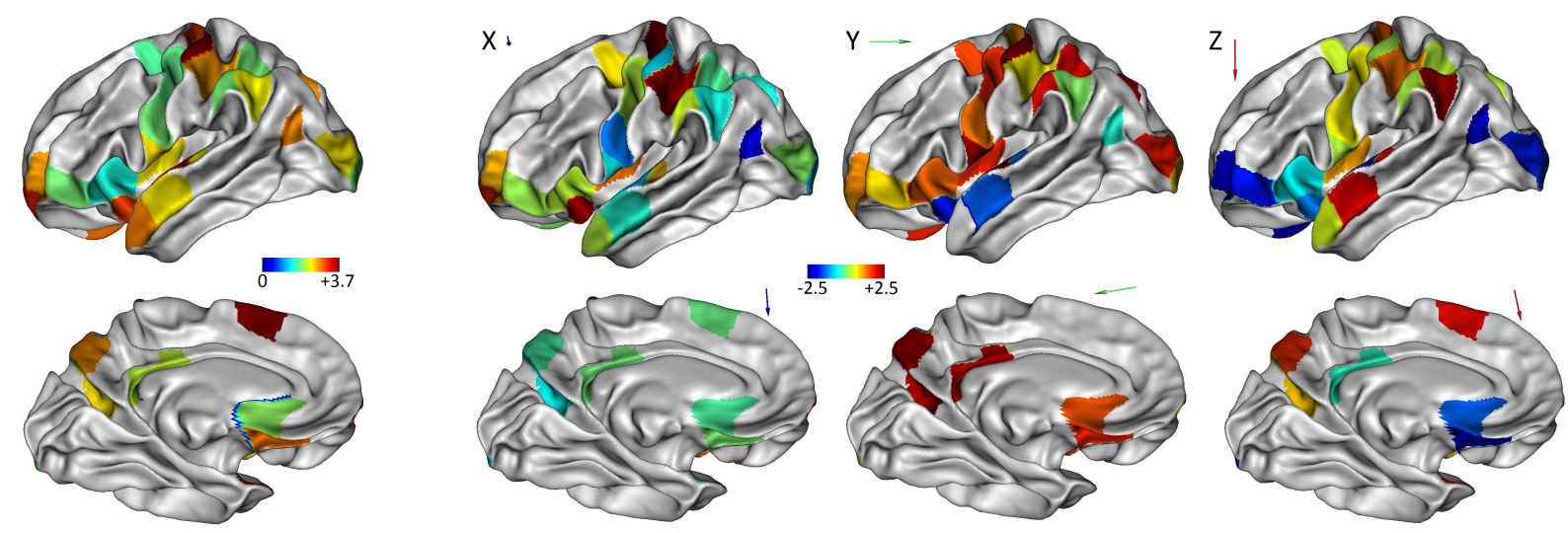

Fig. 4. First order approximation of the pits trajectories in the basins for which temporal variability prevails over inter-subject variability. From left to right, amplitude and projections on the 3 orthogonal axes. Each axis is highlighted by an arrow.

an improvement. Finally, Fig. 4 illustrates for the 32 retained basins the amplitude of the pits trajectories (after first order approximation) and the projections on the $x, y, z$ axes. Pits trajectories from the central sulcus to the occipital lobe evolve in the posterior direction, contrary to the temporal lobe where sulcal basins move anteriorly towards the temporal pole. Pits in the prefrontal lobe evolves in an anterior and dorsal direction.

In this paper, we presented a methodological framework to study pediatric cortical shape development. Our results are preliminary and use only a first order approximation of trajectories, but the full use of the discrete smooth trajectories (Fig. 1), in conjunction with a study of sulcal basins depth, made possible by our pits labelling method, could provide an efficient sparse description of cortical shape development. Another extension could be the study of the temporal development of sulcal basin shape.

\section{ACKNOWLEDGEMENTS}

This work was funded by the Fondation de France (Grant \#2015-00059546). Data presented in this work was obtained from the Cincinnati MR Imaging of NeuroDevelopment (CMIND) database, provided by the Pediatric Functional Neuroimaging Research Network and supported by contract from the Eunice Kennedy Shriver National Institute of Child Health and Human Development (HHSN275200900018C).

\section{REFERENCES}

[1] S. Takerkart, G. Auzias, L. Brun, and O. Coulon, "Structural graph-based morphometry: A multiscale searchlight framework based on sulcal pits," Medical Image Analysis, vol. 35, pp. 32-45, 2017.

[2] K. Im, N.M. Raschle, S.A. Smith, P.E. Grant, and N. Gaab, "Atypical sulcal pattern in children with devel- opmental dyslexia and at-risk kindergarteners," Cerebral Cortex, vol. 26(3), pp. 1138-48, 2015.

[3] Y. Meng, G. Li, W. Lin, J.H. Gilmore, and D. Shen, "Spatial distribution and longitudinal development of deep cortical sulcal landmarks in infants," NeuroImage, vol. 100, pp. 206-218, 2014.

[4] L. Brun, G. Auzias, M. Viellard, N. Villeneuve, N. Girard, ..., and C. Deruelle, "Localized misfolding within brocas area as a distinctive feature of autistic disorder," Biological Psychiatry: Cognitive Neuroscience and Neuroimaging, vol. 1(2), pp. 160-168, 2016.

[5] G. Auzias, L. Brun, C. Deruelle, and O. Coulon, "Deep sulcal landmarks: Algorithmic and conceptual improvements in the definition and extraction of sulcal pits," NeuroImage, vol. 111, pp. 12-25, 2015.

[6] K. Im and H.J. Jo, "Spatial distribution of deep sulcal landmarks and hemispherical asymmetry on the cortical surface," Cerebral Cortex, vol. 20(3), pp. 107786, 2010.

[7] K. Im, R. Pienaar, J.M. Lee, J.K. Seong, Y.Y. Choi, K.H. Lee, and P.E. Grant, "Quantitative comparison and analysis of sulcal patterns using sulcal graph matching: A twin study," NeuroImage, vol. 57(3), pp. 1077-86, 2011.

[8] M. Boucher, S. Whitesides, and A. Evans, "Depth potential function for folding pattern representation, registration and analysis," Medical Image Analysis, vol. 13(2), pp. 203-214, 2009.

[9] N. Charon and A. Trouvé, "The varifold representation of nonoriented shapes for diffeomorphic registration," SIAM J. Imaging Sci., vol. 6(4), pp. 2547-80, 2010.

[10] I. Kaltenmark, B. Charlier, and N. Charon, "A general framework for curve and surface comparison and registration with oriented varifolds," The IEEE Conference on Computer Vision and Pattern Recognition, 2017. 